\title{
Hair Growth Promotion by $\delta$-Opioid Receptor Activation
}

\author{
Mei Zheng', Nahyun Choi ${ }^{1}$, Gianfranco Balboni ${ }^{2}$, Ying Xia ${ }^{3}$ and Jong-Hyuk Sung ${ }^{1,4, *}$ \\ ${ }^{1}$ STEMORE Co. Ltd., Incheon 21983, Republic of Korea \\ ${ }^{2}$ Department of Life and Environment Sciences, University of Cagliari, Cagliari 09124, Italy \\ ${ }^{3}$ Shanghai Key Laboratory of Acupuncture Mechanism and Acupoint Function, Fudan University, Shanghai 200032, China \\ ${ }^{4}$ College of Pharmacy, Institute of Pharmaceutical Sciences, Yonsei University, Incheon 21983, Republic of Korea
}

\begin{abstract}
Literature has revealed that the delta opioid receptor (DOR) exhibited diverse pharmacological effects on neuron and skin. In the present study, we have investigated whether the activation of DOR has hair-growth promotion effects. Compared with other opioid receptor, DOR was highly expressed in epidermal component of hair follicle in human and rodents. The expression of DOR was high in the anagen phase, but it was low in the catagen and telogen phases during mouse hair cycle. Topical application of UFP512 , a specific DOR agonist, significantly accelerated the induction of the anagen in $\mathrm{C}_{3} \mathrm{H}$ mice. Topical application of UFP-512 also increased the hair length in hair organ cultures and promoted the proliferation and the migration of outer root sheath (ORS) cells. Similarly, pharmacological inhibition of DOR by naltrindole significantly inhibited the anagen transition process and decreased hair length in hair organ cultures. Thus, we further examined whether Wnt/ $\beta$-catenin pathway was related to the effects of DOR on hair growth. We found that Wnt/ $\beta$-catenin pathway was activated by UFP-512 and siRNA for $\beta$-catenin attenuated the UFP-512 induced proliferation and migration of ORS cells. Collectively, result established that DOR was involved in hair cycle regulation, and that DOR agonists such as UFP-512 should be developed for novel hair-loss treatment.
\end{abstract}

Key Words: $\delta$-opioid receptor, UFP-512, Outer root sheath cells, Hair growth, Anagen, Wnt/ß-catenin

\section{INTRODUCTION}

UFP-512, an agonist of the delta opioid receptor (DOR), is known to exhibit diverse pharmacological effects on neuron (He et al., 2013; Sheng et al., 2018). UFP-512 was initially developed for treatment of mood disorder (Aguila et al., 2007) and shown to elicit robust anxiolytic- and antidepressant-like effects in rodents (Vergura et al., 2008). Subsequent studies have demonstrated other beneficial effects of UFP-512. For example, UFP-512 activated the motor activity in hemiparkinsonian rats by controlling the nigro-thalamic pathway (Mabrouk et al., 2009). The activation of the DOR by UFP-512 induced the protection of cortical cells against hypoxic/ischemic insults (Yang et al., 2012) and protected cells against oxidative damages by inducing Nrf2 (Cao et al., 2015). The activation of DOR also inhibited the production of inflammatory cytokines during neonatal hypoxic-ischemic encephalopathy (Qiu et al., 2019). However, whether UFP-512 might possess the hair growth-promoting effect is unknown at present.

There exist three types of opioid receptor: the mu $(\mu)$ opi- oid receptor (MOR), the delta $(\delta)$ opioid receptor (DOR), and the kappa ( $\kappa)$ opioid receptor (KOR). These opioid receptors are highly homologous in protein sequence and belong to the family of the G protein-coupled receptors (GPCRs) (Zaki et al., 1996). Although the expression and the function of opioid receptors are poorly characterized in the skin and the hair, the role of opioids in protecting the skin and regenerating the hair growth was previously recognized. For example, opioids accelerated the wound healing by upregulating the expression of endothelial and inducible nitric oxide synthase and the vascular endothelial-derived growth factor receptor, Flk1 (Poonawala et al., 2005). Morphine also stimulated the migration of oral epithelial cells via the activation of DOR (Charbaji et al., 2012). Consistent with these observations, the genetic deletion of DOR altered skin differentiation and delayed wound healing (Bigliardi-Qi et al., 2006), and the activation of the DOR promoted cutaneous wound healing by promoting intercellular adhesion and migration in keratinocytes (Bigliardi et al., 2015). $\beta$-endorphin also stimulated the expression of cytokeratin 16 and downregulated the expression of MOR in

\section{Open Access https://doi.org/10.4062/biomolther.2021.064}

This is an Open Access article distributed under the terms of the Creative Commons Attribution Non-Commercial License (http://creativecommons.org/licenses/by-nc/4.0/) which permits unrestricted non-commercial use, distribution, and reproduction in any medium, provided the original work is properly cited.
Received Apr 1, 2021 Revised May 24, 2021 Accepted May 26, 2021

Published Online Jun 21, 2021

\section{*Corresponding Author}

E-mail: brian99@yonsei.ac.kr

Tel: +82-32-749-4506, Fax: +82-32-209-8239 
human epidermis (Bigliardi-Qi et al., 2000). Notably, topical treatment of the opioid antagonist, low-dose naltrexone (LDN), facilitated the closure of full-thickness wounds in diabetic rats (McLaughlin et al., 2011): LDN is a competitive antagonist of $\mu, \kappa$ and $\gamma$ opioid receptors, and is used for treating alcoholism and opioid addiction. Exposure of LDN at low dosages exhibited a paradoxical effect by increasing the production of endogenous opioids, including $\beta$-endorphins, exhibiting antiinflammatory properties. Therefore, it was proposed that LDN can be an adjunctive treatment for symptomatic alopecia at appropriate dosages (Tortelly et al., 2019).

It is expected that opioid receptors are expressed in epidermal cells, promoting skin regeneration (Neumann et al., 2015; Bigliardi et al., 2016), but the effects of opioid receptors on hair regeneration are unclear. Therefore, we have investigated which types of opioid receptors are expressed in hair follicles and, if so, whether they could promote the hair growth together with the underlying molecular mechanisms.

\section{MATERIALS AND METHODS}

\section{Cell culture}

Human follicle ORS cells, isolated from hair follicles of human scalp skin were cultured in EpiLife ${ }^{\mathrm{TM}}$ Medium with $60 \mu \mathrm{M}$ calcium chloride, 1\% EpiLife ${ }^{\mathrm{TM}}$ Defined Growth Supplement (EDGS) (Thermo Fisher Scientific, Waltham, MA, USA), and $1 \%$ antibiotic-antimycotic (Thermo Fisher Scientific). ORS cells were maintained in a humidified incubator at $37^{\circ} \mathrm{C}$ under $5 \% \mathrm{CO}_{2}$

\section{Cell proliferation assay}

Human ORS cells (passage 3 ) were plated in 48-well plates in triplicates at a density of $6,000-7,000$ cells per well. After $24 \mathrm{~h}$, cell culture medium was replaced with serum-free basal medium containing $1 \%$ anti-antibiotics and exposed to UFP512 for $48 \mathrm{~h}$. In order to measure the rate of cell proliferation, the medium was replaced with prepared CCK-8 solution (Dojindo Molecular Technologies, Rockville, MD, USA), then incubated at $37^{\circ} \mathrm{C}$ under $5 \% \mathrm{CO}_{2}$ for $2 \mathrm{~h}$ and the absorbance was measured at $450 \mathrm{~nm}$ using a microplate reader (Tecan, Männedorf, Switzerland).

\section{Wound healing assay}

The wound healing assay was conducted to determine the migration of ORS cells. In brief, ORS cells (passage $3 ; 5 \times 10^{5}$ cells/ well) were seeded in $35 \mathrm{~mm}$ culture dishes overnight. Confluent ORS cells were wounded using a sterile blue pipette tip, washed with DPBS to remove detached cells, and exposed to vehicle or UFP-512 for $48 \mathrm{~h}$. Cell migration was determined via microscopic examination at five points randomly selected and the horizontal distances of cell migration from the edge of wound were measured.

\section{Real-time quantitative reverse transcription-polymerase chain reaction (real-time quantitative $R T-P C R$ )}

Total RNA was extracted using TRIzol Reagent (Thermo Fisher Scientific), followed by reverse transcription using a cDNA synthesis kit (Nanohelix, Daejeon, Korea). Real-time quantitative reverse-transcription polymerase chain reaction (qRT-PCR) was performed using the StepOne Real-Time PCR System (Thermo Fisher Scientific). GAPDH was used as an internal control to normalize the changes in gene expressions.

\section{Human WNT signaling pathway polymerase chain reaction array}

Gene expression profiles of ORS related with the WNT signaling pathway was analyzed using the Human Transduction Pathway Finder RT² Profiler PCR Array (PAHS-043ZA, Qiagen, Hilden, Germany). Fold changes in expression were calculated using the $\Delta \mathrm{Ct}$ value. Data analysis is based on the ${ }^{\triangle} \mathrm{C} T$ method after normalization of the raw data to housekeeping genes. The red lines indicate \pm 2 folds change in gene expression threshold. The $X$ axis indicates the gene expression changes after vehicle treatment and the $Y$ axis indicates the gene expression changes after treatment of $1 \mu \mathrm{M}$ UFP-512.

\section{Western blotting}

In order to prepare whole cell lysates, adherent ORS cells (passage 3 ) were washed by PBS and immersed in protein extraction solution (Intron, Seoul, Korea) after scraping. Cell lysates were separated by sodium dodecyl sulfate-polyacrylamide gel electrophoresis (SDS-PAGE) using $10 \%$ gels and transferred to PVDF membranes (Millipore, Bedford, MA, USA). Membranes were blocked with $5 \%$ skim milk in TBST (0.1\% Tween-20 in Tris-buffered saline) for $1 \mathrm{~h}$ at room temperature and incubated overnight with primary antibody at $4^{\circ} \mathrm{C}$. At the next day, the membranes were washed three times with TBST and incubated with HRP (horseradish peroxidase)conjugated secondary antibodies for $1 \mathrm{~h}$ at room temperature. The band image of the membrane was acquired by using enhanced chemiluminescence solution (Millipore). Western blotting of $\beta$-actin was conducting to visualize the equal sample loading. The list of primary antibodies used in the present study are illustrated in Table 1.

\section{The induction of anagen in mice}

Mice were maintained and anesthetized according to a protocol approved by Kim et al (2015). This experiment was ap-

Table 1. Primary antibodies used for immunofluorescence analysis and western blot

\begin{tabular}{|c|c|c|c|c|}
\hline Antibodies & Species & Source & Dilution (WB) & Used to identify \\
\hline ERK & Rabbit & Cell Signaling Technology (Danvers, MA, USA) & $1: 1,000$ & ORS cell \\
\hline p-ERK & Mouse & Cell Signaling Technology & $1: 1,000$ & ORS cell \\
\hline p-AKT & Rabbit & Cell Signaling Technology & $1: 1,000$ & ORS cell \\
\hline${ }^{\mathrm{S} 552} \beta$-catenin & Rabbit & Cell Signaling Technology & $1: 1,000$ & ORS cell \\
\hline$\beta$-catenin & Rabbit & Cell Signaling Technology & $1: 1,000$ & ORS cell \\
\hline$\beta$-actin & Mouse & Santa Cruz Biotechnology (Santa Cruz, CA, USA) & $1: 2,000$ & ORS cell \\
\hline
\end{tabular}


proved by the Institutional Animal Care and Use Committee of Yonsei University (Seoul, Korea) (IACUC120002). In brief, the dorsal area $(2.5 \mathrm{~cm} \times 4 \mathrm{~cm})$ of 7 week-old $\mathrm{C}_{3} \mathrm{H} / \mathrm{HeN}$ male mice in the telogen stage of the hair cycle was shaved using a clipper and electric shaver with a special care taken to avoid the damages in the bare skin. Control or $0.01-0.05 \%$ UFP-512 was topically applied onto the back of mice for 2 weeks and any darkening of the skin, e.g. indicative marker for hair cycle induction, was carefully monitored by photography. At $15^{\text {th }}$ day post-experiment, dorsal hair was shaved and its weight was measured. The number of anagen hair shafts was counted after haematoxylin and eosin (H\&E) staining. UFP-512 was provided by our team members, Prof. Gianfranco Balboni and Prof. Ying Xia.

\section{Skin biopsies}

For depilation experiments, 7-week-old male mice were anesthetized with isoflurane and hairs in a $2.5 \mathrm{~cm} \times 4 \mathrm{~cm}$ area of mid-dorsal skin were manually plucked with wax strips to induce synchronized hair cycling. Hair follicles of mice entering the telogen phase were confirmed by pink to white color change of the skin. Dorsal skin biopsies were taken from euthanized mice by $\mathrm{CO}_{2}$ inhalation. Hairs on the back of mice were carefully shaved using an electric clipper before harvesting skin biopsies. Collected skin tissues were processed for preparation of paraffin block.

\section{Primary hair organ culture}

The hair growth promoting activity of mouse vibrissae was observed by using primary hair organ culture. Hair follicles (HFs) were isolated and cultured according to the method previously described by Jindo and Tsuboi (Jindo et al., 1994). Normal vibrissal HFs in the anagen phase were obtained from the upper lip region of mice using a scalpel and tweezers. Isolated murine vibrissal HFs were placed in a defined medium (serum-free Williams E medium supplemented with $2 \mathrm{mM} \mathrm{L-}$ glutamine, $10 \mu \mathrm{g} / \mathrm{mL}$ insulin, $10 \mathrm{ng} / \mathrm{mL}$ hydrocortisone, $100 \mathrm{U} /$ $\mathrm{mL}$ penicillin, and $100 \mu \mathrm{g} / \mathrm{mL}$ streptomycin) and exposed to vehicle or UFP-512 at various concentrations. Individual HFs were photographed $72 \mathrm{~h}$ after the start of incubation, using (Edmund Optics Ltd, UK) and the changes in hair length were calculated based on the images. Values are expressed as mean \pm standard error (SE) of 10-12 vibrissal HFs.

Table 2. The human specific primer sequences

\begin{tabular}{|c|c|c|}
\hline \multirow[t]{2}{*}{$\beta$-catenin } & Forward primer & 5 -CGUUCUCCUCAGAUGGUGU-3` \\
\hline & Reverse primer & $5^{\circ}$-ACACCAUCUGAGGAGAACG-3` \\
\hline \multirow[t]{2}{*}{ DOR } & Forward primer & $5^{\circ}$-CCAAGGCCAAGCUGAUCAA-3` \\
\hline & Reverse primer & $5^{\circ}$-UUGAUCAGCUUGGCCUUGG-3` \\
\hline
\end{tabular}

\section{Small interfering RNA (siRNA) transfection}

Cells were seeded in $35 \mathrm{~mm}$ culture dish at a density of $5 \times 10^{4}$ cells/well and allowed to reach approximately $60 \%$ confluence on the day of transfection. siRNA constructs were ordered from Bioneer corporation (Daejeon, Korea) and their sequences are illustrated in Table 2. Cells were transfected with 20 nM siRNA using Lipofectamine ${ }^{\mathrm{TM}}$ RNAiMAX Transfection Reagent (Thermo Fisher Scientific) according to the manufacturer's instructions.

\section{Immunofluorescence staining in vitro}

Human ORS cells were fixed with formaldehyde. After washing with $1 \mathrm{x}$ PBS, cells were exposed to primary antibodies overnight at $4^{\circ} \mathrm{C}$. Cells were washed three times with $1 \mathrm{x}$ PBS and stained with Alexa Fluor 488-goat anti-rabbit IgG for $1 \mathrm{~h}$ or with 4,6-diamidino-2-phenylindole (DAPI) (Sigma-Aldrich, St. Louis, MO, USA) for visualizing the nucleus. The immunofluorescent images was acquired using a Zeiss LSM700 confocal microscope (Ca Zeiss, Oberkochen, Germany). Primary antibodies used in this study are presented in Table 3.

\section{Immunofluorescence staining in vivo}

Human HFs were kindly received from Prof. Sang Ho Oh (Severance Hospital, Yonsei, University College of Medicine, Seoul, Korea). Unstained paraffin slides were deparaffinized and the antigen retrieval was performed using citrate buffer (Scy Tek, Logan, UT, USA). Slides were then blocked with $1 \%$ bovine serum albumin (BSA) in 1x PBS for $1 \mathrm{~h}$ at RT before the application of primary antibodies overnight at $4^{\circ} \mathrm{C}$. After the slides were washed with $0.05 \%$ Triton-PBS for 3 times, the slides stained with Alexa Fluor 488-goat anti-rabbit lgG or Alexa Fluor-594 goat anti-mouse IgG (Invitrogen, Carlsbad, CA, USA) for $1 \mathrm{~h}$ at RT with DAPI. The immunofluorescent images were acquired using a Zeiss LSM700 confocal microscope (Ca Zeiss). Primary antibodies used in this study are presented in Table 3.

\section{Statistical analysis}

All data are expressed as the mean \pm standard deviation (SD) of three independent experiments. Statistical differences were evaluated using one-way ANOVA or Student's t-test.

\section{RESULTS}

\section{Delta opioid receptor (DOR) is dominantly expressed in hair follicles}

We first examined the expression levels of DOR, MOR, and KOR in human and mouse hair fibroblasts (HFs) by immunofluorescence staining. As a result, we observed that DOR, but not MOR and KOR, was selectively expressed in the ORS

Table 3. Primary antibodies used for immunofluorescence staining in vivo

\begin{tabular}{lllcl}
\hline Antibodies & Species & \multicolumn{1}{c}{ Source } & Dilution (IF) & Used to identify \\
\hline DOR & Rabbit & Abcam (Cambridge, MA, USA) & $1: 200$ & Hair follicle \& ORS cell \\
MOR & Rabbit & Invitrogen & $1: 200$ & Hair follicle \\
KOR & Rabbit & Prosci (San Diego, CA, USA) & $1: 200$ & Hair follicle \\
S363DOR & Rabbit & Biorbyt (Cambridge, GB, UK) & $1: 200$ & Hair follicle \& ORS cell \\
Keratin 17 & Mouse & Santa cruz & $1: 100$ & Hair follicle \\
\hline
\end{tabular}


A

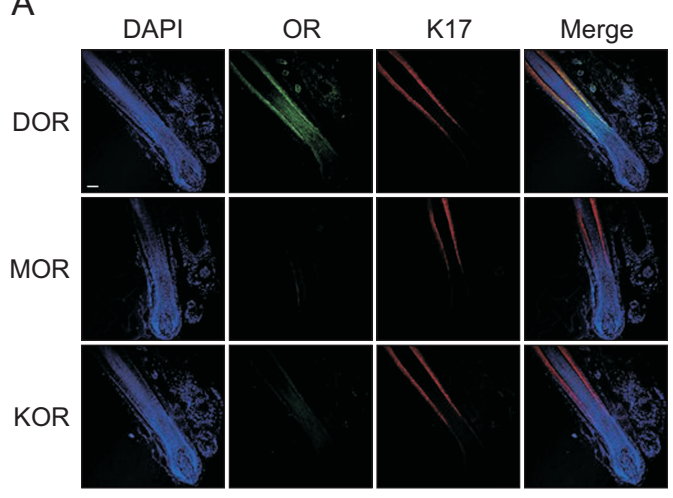

B

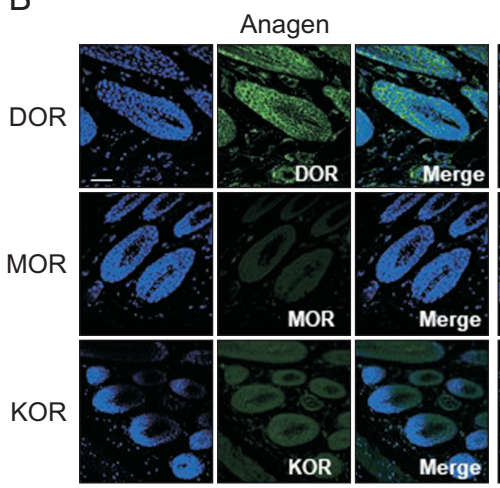

Telogen

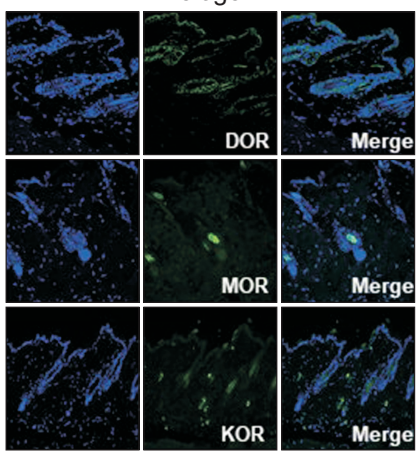

Fig. 1. Delta opioid receptor expression in the hair follicle. (A) Immunostaining showed that among the 3 opioid receptors, only DOR (green) highly expressed and localized in the ORS region of HFs and co-localized with keration 17 (k12, red, an ORS marker) in the HF. (B) DOR was detected in the HFs in the anagen phase, and weakly expressed in the hair germ and bulge area during telogen phase. MOR and KOR were difficultly detected in the HFs in both anagen and telogen phase. DAPI staining (blue) indicates nuclei.

A

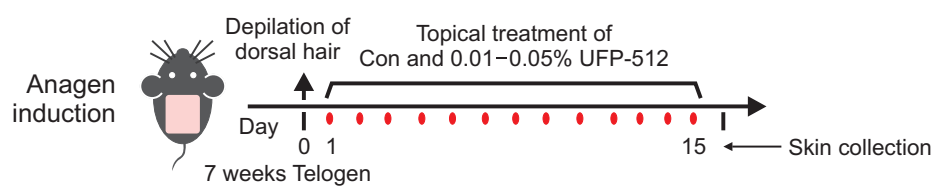

B

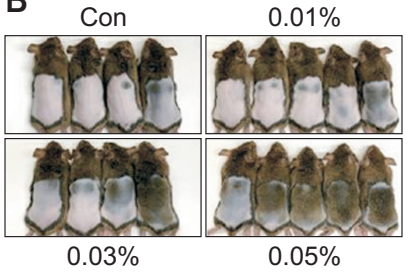

$E$

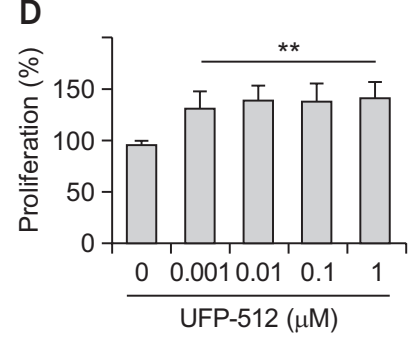

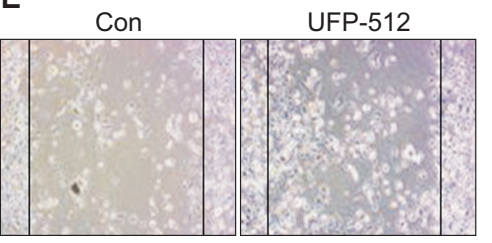
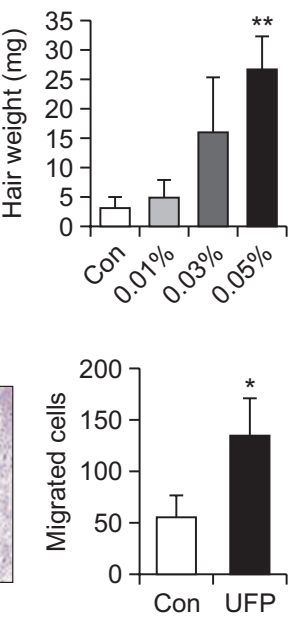

Fig. 2. Hair growth-promoting effects of UFP-512. (A) The experiment design. The back skin of 7-week-old $\mathrm{C}_{3} \mathrm{H}$ mice was shaved, and $0.01 \%-0.05 \%$ UFP-512 was topically applied. (B) Compared with the negative control, topical application of UFP-512 facilitated the telogento-anagen transition in $\mathrm{C} 3 \mathrm{H}$ mice at day 15. Dorsal hair weight was measured. ${ }^{* *} p<0.01$. (C) UFP-512 treatment promoted mouse vibrissa follicle growth ex vivo. * $p<0.05, n=10$ mouse vibrissae follicle per group. The proliferation (D) and migration (E) of hORS cells was measured. ${ }^{*} p<0.05,{ }^{* *} p<0.01$.

region of human scalp HFs: keratin 17 (k17) was costained as ORS marker 17 (Fig. 1A). Similarly, DOR was selectively expressed in ORS cells of mice HFs during the anagen phase and its level decreased during the telogen phases (Fig. 1B). Together, these results suggest that DOR might be associated with the regulation of hair cycling.

\section{UFP-512 induced the growth of mouse hair, and the proliferation and the migration of hORS cells}

We used 7 week-old male $\mathrm{C}_{3} \mathrm{H} / \mathrm{HeN}$ mice in the telogen phase to investigate the telogen-to-anagen transition by UFP512 (Fig. 2A). As a result, we observed that topical application of UFP-512 (0.01\%-0.05\%) facilitated the telogen-to-anagen transition in $\mathrm{C}_{3} \mathrm{H} / \mathrm{HeN}$ mice at day 15 in a dose-dependent manner (Fig. 2B). We also evaluated the hair growth-promot- ing effect of UFP-512 in murine vibrissa organ cultures and found that UFP-512 increased the growth of hair in murine vibrissae ex vivo (Fig. $2 \mathrm{C}$ ). In addition, we observed that UFP512 significantly promoted the proliferation (Fig. 2D) and migration of hORS cells (Fig. 2E).

\section{The inhibition of DOR attenuated the hair growth and hORS cell proliferation}

Because of a high expression of DOR of HFs in the anagen phase than the telogen phase, it can be assumed that DOR might be associated with the regulation of the hair-growth cycle. Supporting for this notion, we observed that subcutaneous injection of UFP-512 significantly increased DOR phosphorylation of HFs in the anagen phase (Fig. $3 A$ ) and cultured hORS cells (Fig. 3B). Next, we used siRNA to decrease the 
A

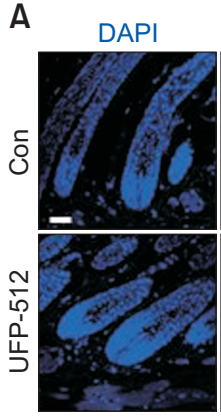

DAPI/p-DOR

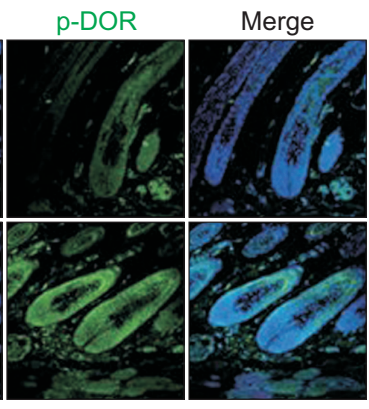

Scale bar $=50 \mu \mathrm{m}$
B

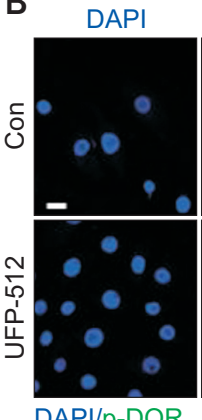

$\mathrm{DAPI} / \mathrm{p}-\mathrm{DOR}$

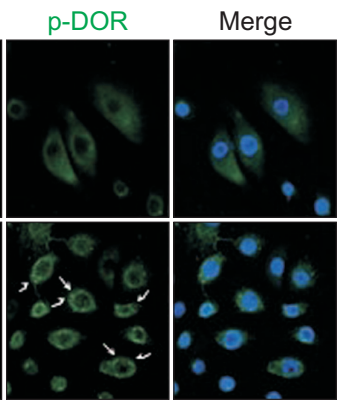

Scale bar $=50 \mu \mathrm{m}$
C

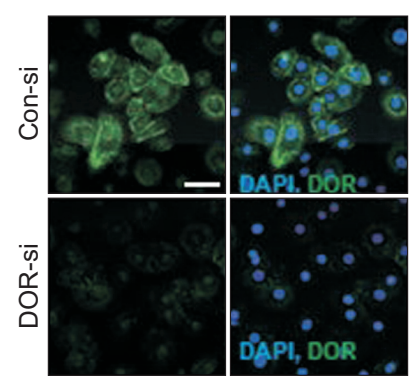

$\mathbf{F}$

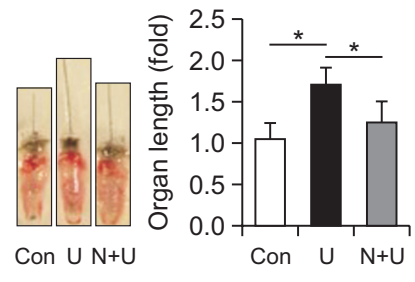

Fig. 3. Activation of DOR in hair follicle and ORS cells. (A) 4-week-old $\mathrm{C}_{3} \mathrm{H}$ mice was used. Phosphorylation of DOR was detected at 30 min after $1 \mu \mathrm{M}$ UFP-512 subcutaneous injection into the dorsal skin of shaved mice. Increased p-DOR was observed in matrix and ORS cells at anagen HFs of UFP-512 treated mice. Scale bar=50 $\mu \mathrm{m}$. (B) DOR was activated and phosphorylated by $1 \mu \mathrm{M}$ of UFP-512 in hORS cells (as indicated by the arrow in immunostaining). Scale bar=20 $\mu \mathrm{m}$. DAPI staining (blue) indicates nuclei. (C, D) Proliferation was monitored in con- and DOR-siRNA treated hORS cells. DOR knockdown was achieved using siRNA transfection and immunostaining. ${ }^{* *} p<0.01$. (E) Compared with the negative control, topical application of naltrindole (0.01\%-0.05\%) significantly delay the telogen-to-anagen transition in $\mathrm{C}_{3} \mathrm{H}$ mice. Dorsal hair weight was measured. ${ }^{* *} p<0.01$. (F) UFP-512 induced hair growth was attenuated by Naltrindole. ${ }^{*} p<0.05$.

expression level of DOR in cultured hORS cells (Fig. 3C), Results also showed a UFP-512-induced increase in the proliferation of ORS cells that was reduced by DOR siRNA (Fig. 3D). In addition, DOR pharmacological inhibition was tested. The specific antagonist, naltrindole, attenuated UFP-512-induced hair growth in mice vibrissa organ culture. Furthermore, 7 week-old male $\mathrm{C}_{3} \mathrm{H} / \mathrm{HeN}$ mice in the telogen phase was used to investigate the telogen-to-anagen transition by naltrindole. Compared with the negative control, topical application of naltrindole $(0.01 \%, 0.05 \%)$ significantly delayed the telogento-anagen transition in $\mathrm{C}_{3} \mathrm{H} / \mathrm{HeN}$ mice (Fig. 3E). Naltrindole significantly inhibited the hair weight in a dose-dependent manner as well (Fig. 3F). These results imply that both protein level and activity of DOR are important for regulating the hairgrowth cycle.

\section{UFP-512 regulated Wnt/ $\boldsymbol{\beta}$-catenin signaling in hORS cells}

We next explored the underlying mechanism of hair growth promotion by UFP-512 in cultured hORS cells, as studies have demonstrated that DOR signaling specifically activated the ERK1/2 MAPK pathway in human keratinocyte (Neumann et al., 2015). To confirm this fact, the hORS cells were treated with UFP-512 for 0-120 min. UFP-512 increased phosphorylation of AKT and ERK1/2 (Fig. 4A). Furthermore, UFP-512 also increased active $\beta$-catenin (ser552) expression, which protects its degradation from GSK3 $\beta$ (Aberle et al., 1997).

It has been reported that opioids can activate the canonical Wnt/ $\beta$-catenin signaling pathway (Li et al., 2019). Activation of the Wnt/ $\beta$-catenin pathway is important for HF regeneration and hair shaft growth (Huelsken et al., 2001; Collins et al., 2011). Therefore, we hypothesized the $\mathrm{Wnt} / \beta$-catenin pathway to be involved in UFP-512 induced hair growth. We measured the mRNA levels of the Wnt pathway-related genes using QPCR array after UFP-512 treatment. UFP-512 upregulated the mRNA levels of seven genes (WNT1, WNT2, WNT6, WNT16, WNT11, WISP, and AXIN2) (fold change > 2) among the 84 Wnt pathway-related genes in cultured hORS cells (Fig. 4B, 4C). Also, to investigate whether $\beta$-catenin is involved in UFP-512-regulated proliferation of ORS, we performed a proliferation assay using siRNA for $\beta$-catenin. Results showed that the $\beta$-catenin siRNA treatment decreased UFP-512 induced proliferation of hORS cells (Fig. 4D).

\section{DISCUSSION}

DOR exhibits diverse pharmacological effects on neuron and skin (Bigliardi-Qi et al., 2006). Therefore, we tried to investigate whether DOR activation has hair-growth promotion effects in this study. Compared to other opioid receptors, DOR was highly expressed in epidermal component of hair follicles in humans and rodents. DOR expression was high in anagen and low in catagen and telogen of mice hair-growth cycle. Topical treatment of UFP-512, a specific DOR agonist, significantly accelerated anagen induction in $\mathrm{C}_{3} \mathrm{H}$ mice. UFP512 also increased the hair length in hair organ cultures and enhanced proliferation as well as migration of hORS cells and increased the phosphorylation of DOR in hair follicles and hORS cells as well. Alternatively, pharmacological inhibition of DOR by naltrindole significantly delayed anagen transition and decreased hair length in hair organ culture as well. We also found that Wnt/ $\beta$-catenin pathway was activated by UFP- 
A
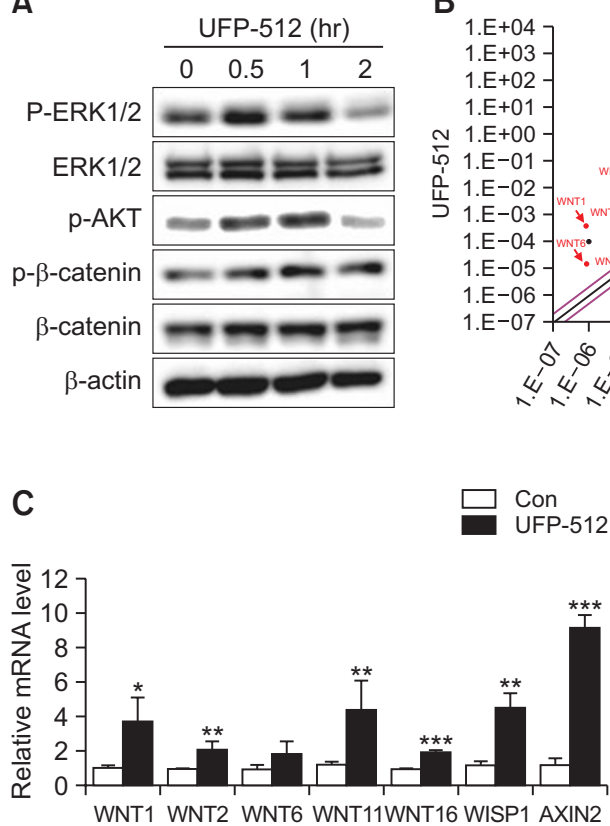

B

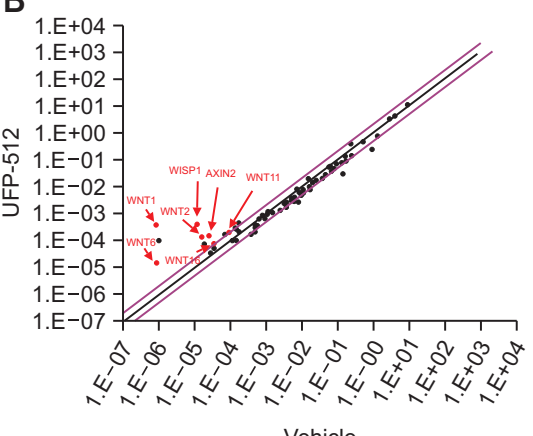

Vehicle

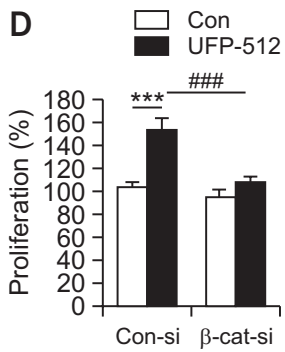

Fig. 4. Involvement of AKT, MAPK/ERK and Wnt/ $\beta$-catening signaling pathway. (A) AKT and MAPK/ERK signaling pathways were assessed under UFP-512 treatment using western blot. (B) Wnt signaling pathway was observed using a $\mathrm{RT}^{2}$ profiler PCR assay. Among the 84 Wnt-related genes, seven genes were increased (WNT1, WNT2, WNT6, WNT16, WNT11, WISP, and AXIN2). (C) qRT-PCR was used to confirm upregulation of mRNA expression. ${ }^{*} p<0.05$, ${ }^{* *} p<0.01$, ${ }^{* * *} p<0.001$. (D) Involvement of $\beta$-catenin in UFP-512 induced proliferation of ORS was observed using $\beta$-catenin siRNA transfection. ${ }^{* * *} p<0.001$ vs. cells exposed to con; $\&{ }^{\# \#} p<0.001$ vs. $\beta$-cat-si/UFP-512 treated cell to con-si/UFP-512.

512, and siRNA for $\beta$-catenin attenuated the UFP-512 induced proliferation of hORS cells. Collectively, results showed that DOR was involved in hair-growth cycle regulation, and DOR agonists exhibit hair-growth promoting effects.

Literature has shown that $\beta$-endorphin, one of the prominent endogenous opioid receptor ligands, was expressed in the skin of C57BL/6 mouse, and accumulated in the pilosebaceous apparatus during the hair-growth cycle (Furkert et al., 1997). They also demonstrated that anagen-associated increase in $\beta$-endorphin concentration and its decline during the catagen/telogen phase raise the possibility of a regulatory function of DOR in cyclic changes of hair follicles. However, they did not examine the opioid receptor expression and functional involvement. We first investigated in this study, which receptor was expressed and involved in hair-growth progression. Among the subtype of opioid receptors, only DOR was predominantly expressed in human and mouse hair follicles. We also measured the expression of DOR during the anagen to telogen phase in $\mathrm{C}_{3} \mathrm{H}$ mice. Interestingly, DOR was highly expressed in ORS and matrix cells during hair-growth period (anagen phase) compared with hair regress period (telogen phase). Collectively, these indicate that DOR plays a key role in hair-growth, and is involved in the regulation of hair-growth cycle (Fig. 1).

UFP-512 was shown to behave in vitro as a selective and potent DOR agonist and to promote antidepressant- and anxiolytic-like effects in vivo (Vergura et al., 2008). UFP-512 has been demonstrated up to 1000 -fold more potent to compete $\left[{ }^{3} \mathrm{H}\right]$-diprenorphine for hDOR binding than other DOR selective and nonselective opioid agonists (Polastron et al., 1994; Marie et al., 2003), and displayed a high affinity for the DOR that was associated with a high selectivity over MOR (160-fold) and KOR (3500-fold) sites. We find that UFP-512 induced ORS proliferation, migration, and increased HF length in the mice organ cultures. We also demonstrated for the first time that UFP-512 accelerated the telogen-to-anagen transition in $\mathrm{C}_{3} \mathrm{H}$ mice (Fig. 2). Phosphorylation of DOR was also increased by subcutaneously injection of UFP-512 in 4-week-old $\mathrm{C}_{3} \mathrm{H} / \mathrm{HeN}$ mice. In addition, blockade of DOR by transfection of DORsiRNA or treatment with naltrindole (a specific antagonist of DOR) decreased UFP-512-induced hair growth in a telogento-anagen transition model. Collectively, these indicate that DOR agonist should be a new target for development as an anti-hair-loss drug.

The Wnt/ $\beta$-catenin pathway regulates cell proliferation and is important for the hair-growth cycle regulation, hair morphogenesis, and hair regeneration (Andl et al., 2002; Ito et al., 2007). Epidermal Wnt is required for activation of $\beta$-catenin signaling pathway, not only within the epidermis, but also across the epidermis to the dermis (Fu and Hsu, 2013). Forced activation of $\beta$-catenin signaling caused thickening of dermis and enlargement of placodes and dermal condensates in mice, resulting in the initiation of epidermal HFs (Chen et al., 2012). Of note, various opioids could activate the canonical Wnt $/ \beta$ catenin signaling pathway (Wang et al., 2017). For example, agonist-dependent MOR activation modulates Wntless activity, a protein that mediates WNT function by regulating its secretion (Reyes et al., 2012). Therefore, we hypothesized that UFP-512 promoted hair growth via the Wnt signaling pathway. As expected, UFP-512 upregulated the mRNA level of WNT1, WNT2, WNT6, WNT11, WNT16, WISP1, and AXIN2, and increased the active form of $\beta$-catenin (Ser552). Therefore, it 
is reasonable to assume that UFP-512 can stimulate hORS proliferation and promotes hair growth via $W n t / \beta$-catenin signaling pathway activation.

\section{CONFLICT OF INTEREST}

The authors declare no conflict of interest.

\section{ACKNOWLEDGMENTS}

Korean Government and the Tech Incubator Program for Starup Korea (Grant No. 10388970).

\section{REFERENCES}

Aberle, H., Bauer, A., Stappert, J., Kispert, A. and Kemler, R. (1997) Beta-catenin is a target for the ubiquitin-proteasome pathway. EMBO J. 16, 3797-3804.

Aguila, B., Coulbault, L., Boulouard, M., Leveille, F., Davis, A., Toth, G., Borsodi, A., Balboni, G., Salvadori, S., Jauzac, P. and Allouche, S. (2007) In vitro and in vivo pharmacological profile of UFP-512, a novel selective delta-opioid receptor agonist; correlations between desensitization and tolerance. Br. J. Pharmacol. 152, 1312-1324.

Andl, T., Reddy, S. T., Gaddapara, T. and Millar, S. E. (2002) WNT signals are required for the initiation of hair follicle development. Dev. Cell 2, 643-653.

Bigliardi-Qi, M., Bigliardi, P. L., Eberle, A. N., Buchner, S. and Rufli, T. (2000) Beta-endorphin stimulates cytokeratin 16 expression and downregulates mu-opiate receptor expression in human epidermis. J. Invest. Dermatol. 114, 527-532.

Bigliardi-Qi, M., Gaveriaux-Ruff, C., Zhou, H., Hell, C., Bady, P., Rufli, T., Kieffer, B. and Bigliardi, P. (2006) Deletion of delta-opioid receptor in mice alters skin differentiation and delays wound healing. Differentiation 74, 174-185.

Bigliardi, P. L., Dancik, Y., Neumann, C. and Bigliardi-Qi, M. (2016) Opioids and skin homeostasis, regeneration and ageing - what's the evidence? Exp. Dermatol. 25, 586-591.

Bigliardi, P. L., Neumann, C., Teo, Y. L., Pant, A. and Bigliardi-Qi, M. (2015) Activation of the delta-opioid receptor promotes cutaneous wound healing by affecting keratinocyte intercellular adhesion and migration. Br. J. Pharmacol. 172, 501-514.

Cao, S., Chao, D., Zhou, H., Balboni, G. and Xia, Y. (2015) A novel mechanism for cytoprotection against hypoxic injury: delta-opioid receptor-mediated increase in Nrf2 translocation. Br. J. Pharmacol. 172, 1869-1881.

Charbaji, N., Schafer-Korting, M. and Kuchler, S. (2012) Morphine stimulates cell migration of oral epithelial cells by delta-opioid receptor activation. PLoS ONE 7, e42616.

Chen, D., Jarrell, A., Guo, C., Lang, R. and Atit, R. (2012) Dermal betacatenin activity in response to epidermal Wnt ligands is required for fibroblast proliferation and hair follicle initiation. Development 139, 1522-1533.

Collins, C. A., Kretzschmar, K. and Watt, F. M. (2011) Reprogramming adult dermis to a neonatal state through epidermal activation of beta-catenin. Development 138, 5189-5199.

Fu, J. and Hsu, W. (2013) Epidermal Wnt controls hair follicle induction by orchestrating dynamic signaling crosstalk between the epidermis and dermis. J. Invest. Dermatol. 133, 890-898.

Furkert, J., Klug, U., Slominski, A., Eichmuller, S., Mehlis, B., Kertscher, U. and Paus, R. (1997) Identification and measurement of beta-endorphin levels in the skin during induced hair growth in mice. Biochim. Biophys. Acta 1336, 315-322.

He, X., Sandhu, H. K., Yang, Y., Hua, F., Belser, N., Kim, D. H. and Xia, Y. (2013) Neuroprotection against hypoxia/ischemia: delta-opioid receptor-mediated cellular/molecular events. Cell. Mol. Life Sci.
70, 2291-2303.

Huelsken, J., Vogel, R., Erdmann, B., Cotsarelis, G. and Birchmeier, W. (2001) Beta-Catenin controls hair follicle morphogenesis and stem cell differentiation in the skin. Cell 105, 533-545.

Ito, M., Yang, Z., Andl, T., Cui, C., Kim, N., Millar, S. E. and Cotsarelis, G. (2007) Wnt-dependent de novo hair follicle regeneration in adult mouse skin after wounding. Nature 447, 316-320.

Jindo, T., Tsuboi, R., Imai, R., Takamori, K., Rubin, J. S. and Ogawa, H. (1994) Hepatocyte growth factor/scatter factor stimulates hair growth of mouse vibrissae in organ culture. J. Invest. Dermatol. 103, 306-309.

Li, Y., Wang, J., Li, Z., Cheng, H., Zhang, Z., Luo, T., Zhang, X., Gao, G., Lu, H. and Li, L. (2019) Propoxyphene mediates oxyhemoglobin-induced injury in rat cortical neurons through up-regulation of active-beta-catenin. Front. Pharmacol. 10, 1616.

Kim, J. H., Park, S. G., Kim, W. K., Song, S. U. and Sung, J. H. (2015) Functional regulation of adipose-derived stem cells by PDGFD. Stem Cells 33, 542-556.

Mabrouk, O. S., Marti, M., Salvadori, S. and Morari, M. (2009) The novel delta opioid receptor agonist UFP-512 dually modulates motor activity in hemiparkinsonian rats via control of the nigro-thalamic pathway. Neuroscience 164, 360-369.

Marie, N., Lecoq, I., Jauzac, P. and Allouche, S. (2003) Differential sorting of human delta-opioid receptors after internalization by peptide and alkaloid agonists. J. Biol. Chem. 278, 22795-22804.

McLaughlin, P. J., Pothering, C. A., Immonen, J. A. and Zagon, I. S. (2011) Topical treatment with the opioid antagonist naltrexone facilitates closure of full-thickness wounds in diabetic rats. Exp. Biol. Med. (Maywood) 236, 1122-1132.

Neumann, C., Bigliardi-Qi, M., Widmann, C. and Bigliardi, P. L. (2015) The delta-opioid receptor affects epidermal homeostasis via ERKdependent inhibition of transcription factor POU2F3. J. Invest. Dermatol. 135, 471-480.

Polastron, J., Mur, M., Mazarguil, H., Puget, A., Meunier, J. C. and Jauzac, P. (1994) SK-N-BE: a human neuroblastoma cell line containing two subtypes of delta-opioid receptors. J. Neurochem. 62, 898-906.

Poonawala, T., Levay-Young, B. K., Hebbel, R. P. and Gupta, K. (2005) Opioids heal ischemic wounds in the rat. Wound Repair Regen. 13, 165-174.

Qiu, J., Chao, D., Sheng, S., Khiati, D., Zhou, X. and Xia, Y. (2019) Delta-opioid receptor-Nrf-2-mediated inhibition of inflammatory cytokines in neonatal hypoxic-ischemic encephalopathy. Mol. Neurobiol. 56, 5229-5240.

Reyes, B. A., Vakharia, K., Ferraro, T. N., Levenson, R., Berrettini, W. H. and Van Bockstaele, E. J. (2012) Opiate agonist-induced redistribution of Wntless, a mu-opioid receptor interacting protein, in rat striatal neurons. Exp. Neurol. 233, 205-213.

Sheng, S., Huang, J., Ren, Y., Zhi, F., Tian, X., Wen, G., Ding, G. Xia, T. C., Hua, F. and Xia, Y. (2018) Neuroprotection against hypoxic/ischemic injury: delta-opioid receptors and BDNF-TrkB pathway. Cell. Physiol. Biochem. 47, 302-315.

Tortelly, V. D., De Mattos, T., Fernandes, L. S. A., Nunes, B. E. M. and Melo, D. F. (2019) Low-dose naltrexone: a novel adjunctive treatment in symptomatic alopecias? Dermatol. Online J. 25, 13030/ qt6j45h81f.

Vergura, R., Balboni, G., Spagnolo, B., Gavioli, E., Lambert, D. G., McDonald, J., Trapella, C., Lazarus, L. H., Regoli, D., Guerrini, R., Salvadori, S. and Calo, G. (2008) Anxiolytic- and antidepressantlike activities of H-Dmt-Tic-NH-CH(CH2-COOH)-Bid (UFP-512), a novel selective delta opioid receptor agonist. Peptides 29, 93-103.

Wang, J., Zhu, G., Huang, L., Nie, T., Tao, K., Li, Y. and Gao, G. (2017) Morphine administration induces change in anxiety-related behavior via Wnt/beta-catenin signaling. Neurosci. Lett. 639, 199-206.

Yang, Y., Zhi, F., He, X., Moore, M. L., Kang, X., Chao, D., Wang, R., Kim, D. H. and Xia, Y. (2012) Delta-opioid receptor activation and microRNA expression of the rat cortex in hypoxia. PLOS ONE 7, e51524.

Zaki, P. A., Bilsky, E. J., Vanderah, T. W., Lai, J., Evans, C. J. and Porreca, F. (1996) Opioid receptor types and subtypes: the delta receptor as a model. Annu. Rev. Pharmacol. Toxicol. 36, 379-401. 